\title{
Practical introduction to optical WDM components and systems in student teaching laboratories
}

lain Mauchline, Douglas Walsh, David Moodie, Steve Conner, Walter Johnstone, et al.

lain Mauchline, Douglas Walsh, David Moodie, Steve Conner, Walter Johnstone, Brian Culshaw, "Practical introduction to optical WDM components and systems in student teaching laboratories," Proc. SPIE 9665, Tenth International Topical Meeting on Education and Training in Optics and Photonics, 966510 (3 June 2007); doi: 10.1117/12.2207346

SPIE Event: Tenth International Topical Meeting on Education and Training in Optics and Photonics, 2007, Ottawa, Ontario, Canada 


\title{
Practical Introduction to Optical WDM Components and Systems in Student Teaching Laboratories
}

\author{
Iain Mauchline, Douglas Walsh, David Moodie and Steve Conner \\ OptoSci Ltd, 141 St. James Rd., Glasgow, G4 OLT, Scotland, UK, T: +44 141552 7020, F: +44 141552 3886, E: info@optosci.com
}

Walter Johnstone and Brian Culshaw

EEE Dept., University of Strathclyde, 204 George St., Glasgow, G1 1XW, Scotland, UK

\begin{abstract}
In this paper we describe a new family of teaching packages designed to offer a practical introduction for graduate students of Science and Engineering to the topic of wavelength division multiplexing (WDM) in fibre optics. The teaching packages described here provide students with the background theory before embarking on a series of practical experiments to demonstrate the operation and characterisation of WDM components and systems. The packages are designed in a modular format to allow the user to develop from the fundamentals of fibre optical components through to the concepts of WDM and dense WDM (DWDM) systems and onto advanced topics covering aspects of Bragg gratings. This paper examines the educational objectives, background theory, and typical results for these educational packages.
\end{abstract}

\section{Introduction}

Optical fibre communications has proved to be one of the key application areas, which created, and ultimately propelled the global growth of the photonics industry over the last twenty years. Consequently the teaching of the principles of optical fibre communications has become integral to many university courses covering photonics technology. However to reinforce the fundamental principles and key technical issues students examine in their lecture courses and to develop their experimental skills, it is critical that the students also obtain hands-on practical experience of photonics components, instruments and systems in an associated teaching laboratory. In recognition of this need OptoSci Ltd, in collaboration with academics at Strathclyde and Heriot-Watt Universities, has commercially developed a suite of fully self-contained laboratory based photonics teaching packages for use in universities, colleges, and industrial training centres. This range of packages covering topics from the fundamentals of physical optics through to fibre optic communications, optical network analysis and optical amplifiers has been described in detail previously $[1,2,3,4]$.

In the 1990s, the advent of practical wavelength division multiplexing (WDM) systems revolutionised the fibre optic communications industry by enabling unprecedented increases in data rate over optical fibre. The commercial exploitation of WDM required the development of new components to provide certain required functionality such as multiplexing, demultiplexing and wavelength routing. In addition, existing component technologies had to be adapted to operate to the new specifications required by WDM systems such as the fused fibre biconical taper (FBT) couplers and WDMs used in the EDFAs or the high rejection ratio isolators / circulators used to eliminate feedback to the lasers. In light of this OptoSci Ltd. have designed the ED-WDM series of educator kits to provide students and trainees with a good working knowledge and understanding WDM systems, the components used in them and the measurement techniques used to establish the specifications of these components.

The objectives of the ED-WDM series are to enable students

- to develop a practical understanding and knowledge of the components used in optical networks in general and in WDM networks in particular.

- to acquire a knowledge and understanding of the measurement techniques used to establish component specifications and the practical skills to make these measurements and

- to develop a practical appreciation and understanding of the principles and characteristics of WDM systems.

Tenth International Topical Meeting on Education and Training in Optics and Photonics, edited by Marc Nantel, Proc. of SPIE Vol. 9665, 966510 - (C) 2007 SPIE, OSA, IEEE, ICO doi: $10.1117 / 12.2207346$ 


\section{Design Philosophy}

The overall educational aims of a teaching laboratory are to enable students to consolidate their understanding and knowledge of photonics as presented in an accompanying lecture course and to acquire practical experience of the design, analysis and characteristics of photonics components and systems. To achieve these aims it is essential to take a fully integrated approach to the design of laboratory based photonics teaching packages including the design of dedicated hardware, experimental procedures, exercises and manuals. To ensure that all desirable educational objectives are met and that all of the most important scientific and technical principles, issues and phenomena are addressed, we have developed our suite of fully integrated laboratory based teaching packages in accordance with the following design rules:

- Define the educational objectives in terms of the physical principles, important technical features, design issues and performance characteristics which must be addressed, with particular attention to facilitating student understanding and ability to implement concepts.

- Define the experiments to meet these performance objectives.

- Design the dedicated (custom) hardware to enable the proposed experimental investigation whilst keeping costs within realistic academic teaching budgets.

- Formulate the experimental procedure and manuals to guide the students through the investigation and results analysis (in some cases more open ended investigations may be formulated with minimal guidance to the students).

The primary constraint is cost and the final packages must be affordable within higher education budgets. In general, the packages have been designed as far as possible to be self-contained so that as little ancillary equipment as possible is required. However, where it is advantageous and cost effective to use equipment normally available in student laboratories, the packages have been designed to be compatible with the capabilities of such equipment e.g. a $20 \mathrm{MHz}$ or $50 \mathrm{MHz}$ oscilloscope.

\section{Package Contents}

\subsection{Hardware}

The ED-WDM series is designed in a modular format using the industry standard 19" rack system. The complete version of the kit occupies two 3Ux84HP enclosures, the Optical Components Rack and the Electronics Rack, as shown in Figure 1. Each component part is contained in a standard 3Ux10HP cassette. This modular approach allows the system to be built up from the basics to more advanced levels.
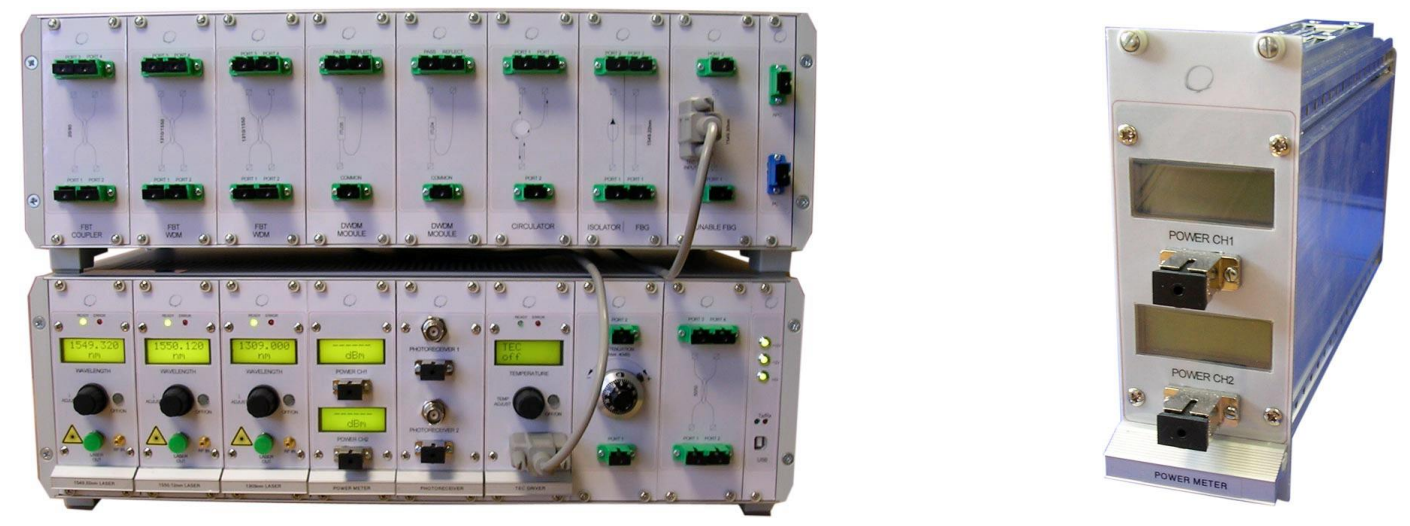

Figure 1: Complete ED-WDM educator kit and individual power meter module

The Optical Components rack is intended to house a range of passive components modules such as couplers, WDMs, an isolator, a circulator and fibre Bragg gratings. Patchcords are provided to allow the external connection between the module as required by the experimental procedures. An SC/APC style of connector is used predominately in the kit to allow easy reconfiguring of the optical set-ups and to minimise spurious backreflections. 
The Electronics Rack is designed to house the lasers, power meters, photoreceivers and TEC controllers along with a variable optical attenuator and 50:50 coupler. This provides the instrumentation to allow the interrogation and investigation of the WDM components and systems. It is also fitted with a USB interface to allow PC control \& monitoring of the instrument by the dedicated driver \& display software supplied with the kit

The electronics modules are described below:-

Laser Diode Modules - self contained units housing a DFB laser and drive electronics. The lasers are set to emit a constant power $(\approx 0 \mathrm{dBm})$ output over a $1-2 \mathrm{~nm}$ range of wavelengths around the specified $\lambda_{\mathrm{c}}$. The operating wavelength of the laser can be adjusted by a $\lambda$-adjust knob on the front panel or by computer control. The operating wavelength is displayed on a LCD display. RF modulation may be applied to the laser via a panel mounted SMB connector.

Power Meter Module - contains twin optical power meters using SC mounted InGaAs photodiodes. These are calibrated in $\mathrm{dBm}$ at $1550 \mathrm{~nm}$ to display incident powers up to a maximum of $+3 \mathrm{dBm}$.

Photoreceiver Module - contains twin wideband (100MHz) photoreceivers using SC mounted InGaAs photodiodes. The maximum recommended peak power to avoid signal distortion is $-8 \mathrm{dBm}$. Fixed attenuators are supplied to allow higher powers to be detected where required. The output from the photodiodes is available from panel mounted BNC connectors.

TEC Driver Module - houses the driver for an external Thermo-Electric-Cooler (TEC) which can be connected via a front panel connector. An LCD displays the Setpoint Temperature in the upper line and the Actual Temperature on the lower. The ON/OFF button can used to re-initialise the TEC in the event of an ERROR condition.

\subsection{Software}

The software provided with the ED-WDM series has three parts:

LVI Plotter - to enable the characterisation of the laser sources by automatically sweeping the drive current of the laser over their operating range and logging the results from the power meter.

$\lambda$-Scan - to perform an automated narrowband wavelength scan $(\sim 2 \mathrm{~nm})$ of the DFB laser, which can be used in conjunction with the power meter modules for spectral characterisation of the DWDM components.

Dispersion_Test - which is used for fibre length and chromatic dispersion measurements

\subsection{Literature}

As with all OptoSci kits the education objective is to provide a comprehensive package to the educator hence extensive literature support is provided. The literature pack for the ED-WDM series is split into three sections:

Laboratory manuals - introducing students to the underlying concepts and architectures of WDM before looking at detail at the components used within such systems. The different technologies used in the realisation of WDM components are then explained and the key operation parameters and limitations identified. This leads onto the experimental exercises which detail the characterisation techniques and procedures required to measure the component performance.

Instructor supplement - containing full sample results and worked examples along with practical notes to assist the instructor.

General Appendices - which contain information on Laser Safety, additional background of DWDM systems such as the ITU grid structure and, crucially, practical tips on aspects of basic handling and care of fibre optic parts which may be necessary for students new to the topic. (This practical tip section has been included in light of feedback from students and instructors, who perhaps had limited experience of fibre optics, experiencing problems with the optical connectors styles and care of fibres.) 


\section{Experimentation}

The ED-WDM series currently addresses four main areas - WDM components, 1310/1550nm WDM systems, DWDM systems and Fibre Bragg gratings. The ED-WDM: WDM Components kit is designed as the basic kit to introduce students to the fundamentals of WDM components and establish the basis of WDM systems. The other topics are addressed by extension modules which provide additional hardware to enable the students to investigate the specific aspects of WDM systems or Bragg gratings. Each of the study areas is described below with examples of the experiments and sample results.

\subsection{WDM Components}

As mentioned above the ED-WDM: WDM Components kit is intended as the starting point of the suite. The kit contains a ITU grid DFB laser $\left(\lambda_{c} \approx 1550 \mathrm{~nm}\right)$, a pair of InGaAs power meters and a variety of standard optical components that are typically present in WDM systems. The components for characterisation include fused fibre couplers, a fuse fibre 1310/1550nm WDM, a micro-optic add-drop multiplexer (OADM) operating at the DFB wavelength, an isolator, a circulator and a fibre Bragg grating.

The kit provides students with the theory and practical ability to study the basics of optical component operation and characterisation. To achieve these objectives the following tasks are carried out:

- Measurement of light, voltage \& current (LVI) characteristics of a DFB laser with operating temperature

- Measurement of insertion loss, directivity and backreflection/return loss for a series of fibre optic components (i.e. coupler, WDM, isolator, circulator, DWDM Mux/Demux devices)

- Determination of isolation / extinction ratios in various optical components

- Examination of narrowband wavelength responses of a number of optical components

\subsubsection{Component Characterisation}

From the theory presented to the students they should have gained an understanding of the background and operation of each component supplied in the package. The experimental process now commences to perform the characterisation of the key physical parameters highlighted in the previous discussion. A standardised approach of presenting the relevant information and detailing the characterisation techniques for each individual component is used in the kit. Each component investigation may then be considered a complete task in itself, allowing the instructor to select which component or components he wishes the students to study.

\subsubsection{Fused Fibre Coupler}

An example of the approach used is presented below for the case of a fused fibre coupler:

\section{A) Define the operating parameters.}

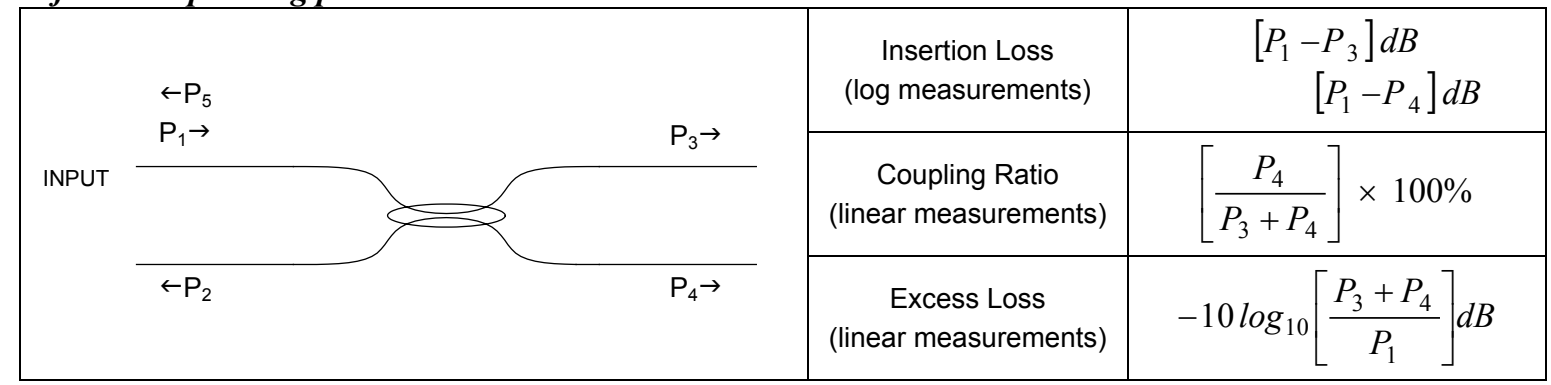

\section{B) Define the experimental set-up required.}

MOWER




\section{C) Detail the measurements required and optical connections that should be made.}

1. Connect the $1550 \mathrm{~nm}$ laser directly to the power meter using the hybrid FC/APC to SC/APC patchcord and measure / note the input power to Port 1 of the coupler. Now connect the hybrid patchcord directly to Port 1 of the Coupler being tested as shown in experimental set-up. Measure the powers emitted from Ports 2, 3 and 4 by connecting them in turn to the Power Meter via standard SC/APC patchcords.

2. Now, connect the $1550 \mathrm{~nm}$ laser to Port 2 and repeat step 1.

\section{D) Analyse the results obtained and comment on the operation of the component being tested.}

Note the measured powers in logarithmic $(\mathrm{dBm})$ and linear form $(\mathrm{mW}$ - calculated from the measured $\log$ values as in Appendix 2). From these measurements calculate values for insertion loss, coupling ratio, and excess loss as detailed above.

Comment on your results.

E) A sample set of results and worked example of the analysis is presented in the Instructor Supplement.

\begin{tabular}{|c|c|c|}
\hline Measurement & $\mathrm{dBm}$ & $\mathrm{mW}$ \\
\hline TEST OUTPUT (Port 1) & -2.75 & 0.531 \\
\hline Port 3 & -4.20 & 0.380 \\
\hline Port 4 & -10.24 & 0.0946 \\
\hline Parameter & Calculation & Result \\
\hline Insertion loss to Port 3 & $(-2.75)-(-4.20) d B$ & $1.45 \mathrm{~dB}$ \\
\hline Insertion loss to Port 4 & $(-2.75)-(-10.24) d B$ & $7.49 \mathrm{~dB}$ \\
\hline Coupling ratio & $\frac{0.0946}{0.380+0.0946} \times 100 \%$ & $19.93 \%$ \\
\hline Excess loss & $-10 \log _{10}\left[\frac{0.380+0.0946}{0.531}\right]$ & $0.48 \mathrm{~dB}$ \\
\hline \multicolumn{3}{|l|}{ Discussion } \\
\hline \multicolumn{3}{|c|}{$\begin{array}{l}\text { From these results the student should report that the coupler has a coupling ratio of } 20 \% \text { at } 1550 \mathrm{~nm} \text {. When the input is } \\
\text { made to Port } 2 \text { of the coupler the outputs should switch i.e. Port } 3 \text { should now the } 20 \% \text { output. }\end{array}$} \\
\hline
\end{tabular}

\subsubsection{Other Components}

A similar process is then followed for each component to examine the relevant parameters as listed below:

\begin{tabular}{||l|l||}
\hline \hline Component & Parameters Examined \\
\hline \hline Fused Fibre WDM & Insertion loss, Wavelength Isolation, Excess Loss \\
\hline Isolator, Circulator & Insertion Loss, Isolation, Return Loss \\
\hline DWDM & Insertion Loss, Wavelength Isolation \\
\hline Fibre Bragg grating & Insertion Loss, Reflectivity \\
\hline
\end{tabular}

\subsubsection{Automated Measurements}

If computer control is available the spectral behaviour of the DWDM components can also be examined using the $\lambda$-scan software program. This is suited to the study of the narrowband components i.e. the OADMs and the Bragg gratings. The set-up shown in Figure 2 allow both arms of the multiplexer to be studied simultaneously using $\lambda$ scan. 


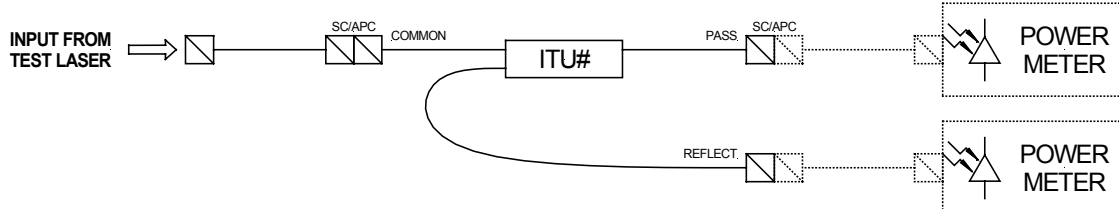

Figure 2: Experimental set-up for spectral behaviour of the OADM

A typical results screen is shown in Figure 3(a), the students are directed save the data sets for further processing to normalise the plot and yield the actual insertion losses to each arm, as displayed in Figure 3(b).

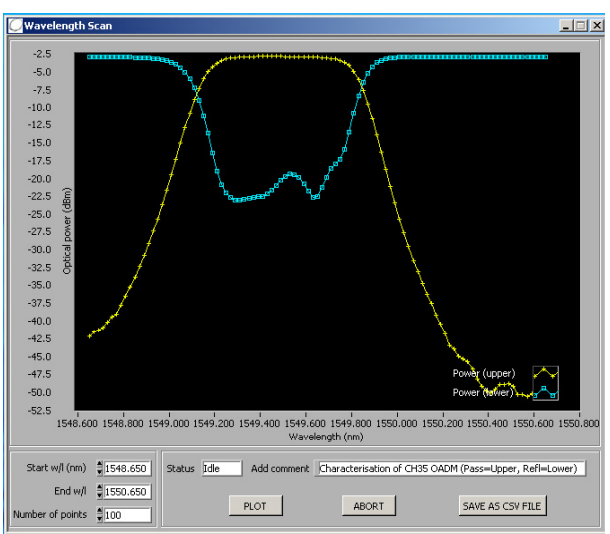

(a)

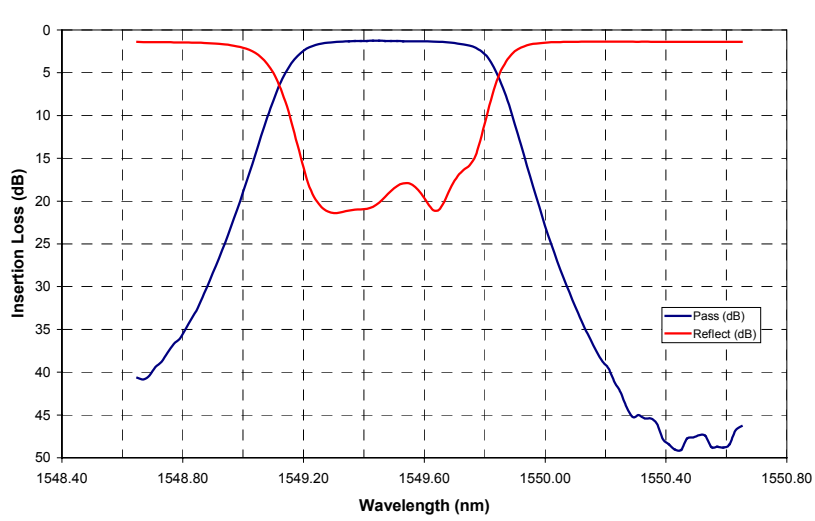

(b)

Figure 3: Spectral characterisation of OADM: (a) Screenshot from $\lambda$-scan and (b) Processed data

The software thus offers a simple way to acquiring the full spectral characteristic of the components, this is especially beneficial when dealing with the very narrow features associated with the fibre Bragg gratings as will been seen later.

\subsubsection{DFB LVI plots}

As one of the most important parts if the WDM systems, the students then carry on to investigate the operation of an DFB laser.

The DFB laser characteristics may be obtained using computer control and the LVI plotting software. Figure 4 shows a typical LVI plotter window from the software and the results of a series of L-I plots for a laser with variation of operating temperature.

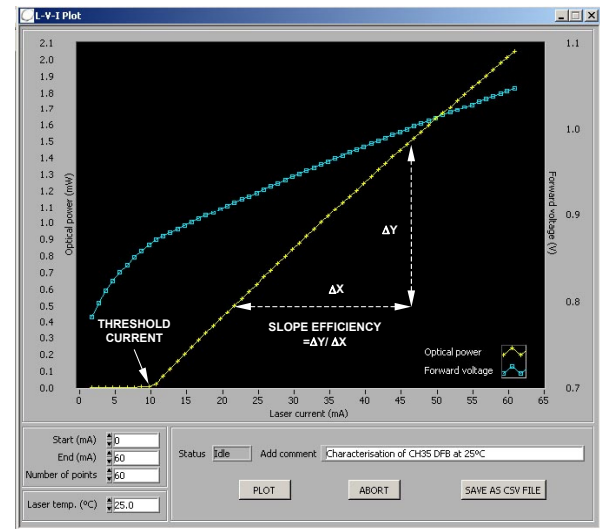

(a)

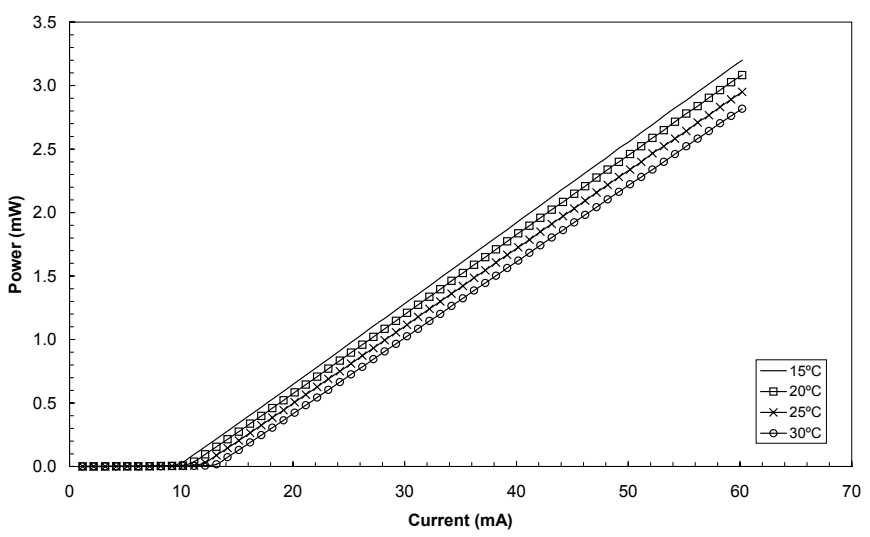

(b)

Figure 4: DFB laser characteristics: (a) LVI plotter window and (b) L-I plots against temperature. 
As the operating temperature of the $1550 \mathrm{~nm}$ laser is increased the threshold current is seen to increase and the slope efficiency decrease. Over this limited temperature range the results show the threshold current temperature dependence is $0.25 \mathrm{~mA} /{ }^{\circ} \mathrm{C}$.

This should highlight to the students the marked temperature dependence of the laser characteristics thus making it imperative to have close control, not only for wavelength stability, but also to provide stable laser output power levels for DWDM systems.

\subsection{0/1550 WDM Systems}

The first of the extension kits expands the fundamentals developed in the components characterisation kit to allow the investigation of practical WDM systems working at $1310 / 1550 \mathrm{~nm}$. The extension includes a second laser $\left(\lambda_{c}=1310 \mathrm{~nm}\right)$, dual photoreceivers and an additional fused fibre 1310/1550nm WDM and a reel of singlemode fibre. To study these WDM systems the students are directed to complete the tasks listed below:

- Measurement of insertion losses and backreflection / return losses for various components supplied with ED-WDM: WDM Components at $1310 \mathrm{~nm}$ and comparison with $1550 \mathrm{~nm}$ measurements.

- Assembly, demonstration and characterisation of a two channel 1310nm \& $1550 \mathrm{~nm}$ WDM system

- Fibre attenuation, length and chromatic dispersion measurements at $1310 \mathrm{~nm} \& 1550 \mathrm{~nm}$.

\subsubsection{Component characterisation at $1310 \mathrm{~nm}$}

By repeating the component characterisation process at $1310 \mathrm{~nm}$ the students will gain an insight into the broadband spectral behaviour of fibre optic components. In the kit a standard coupler and dual-window coupler are supplied to highlight different types that may be encountered, the dual window should show similar operation at both $1310 \mathrm{~nm}$ and $1550 \mathrm{~nm}$ whereas the standard type will operate only as specified at $1550 \mathrm{~nm}$. The micro-optic components (i.e. isolator and circulator) are specified at $1550 \mathrm{~nm}$ hence the students should find operation at $1310 \mathrm{~nm}$ to be outwith the expected values. Most importantly the availability of the second wavelength laser allows the complete characterisation of the fused fibre WDM as shown in Figure 5.

\begin{tabular}{|c|c|c|c|c|c|}
\hline \multirow{4}{*}{ INPUT } & \multirow{4}{*}{$\begin{array}{c}P_{1}\left(\lambda_{3}+\lambda_{4}\right) \\
\rightarrow\end{array}$} & \multirow{3}{*}{ 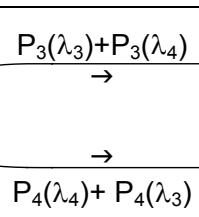 } & \multirow{3}{*}{$\begin{array}{l}\lambda_{3} \text { OUTPUT } \\
\lambda_{4} \text { OUTPUT }\end{array}$} & Insertion Loss $\left(\lambda_{3}\right)$ & {$\left[P_{1}\left(\lambda_{3}\right)-P_{3}\left(\lambda_{3}\right)\right] d B$} \\
\hline & & & & Insertion Loss $\left(\lambda_{4}\right)$ & {$\left[P_{1}\left(\lambda_{4}\right)-P_{4}\left(\lambda_{4}\right)\right] d B$} \\
\hline & & & & Isolation $\left(\lambda_{3}\right)$ & {$\left[P_{3}\left(\lambda_{3}\right)-P_{4}\left(\lambda_{3}\right)\right] d B$} \\
\hline & & & & Isolation $\left(\lambda_{4}\right)$ & {$\left[P_{4}\left(\lambda_{4}\right)-P_{3}\left(\lambda_{4}\right)\right] d B$} \\
\hline
\end{tabular}

Figure 5: 1310/1550nm WDM definitions

From this series of measurements the students are asked to identify suitable connections to obtain the desired multiplexing and demultiplexing operations required for the WDM systems below.

\subsubsection{0/1550 WDM Systems}

The basic characterisation of a simple WDM system shown in Figure 6 is carried out in a similar way to the single component by noting the power levels at either output from each input laser.

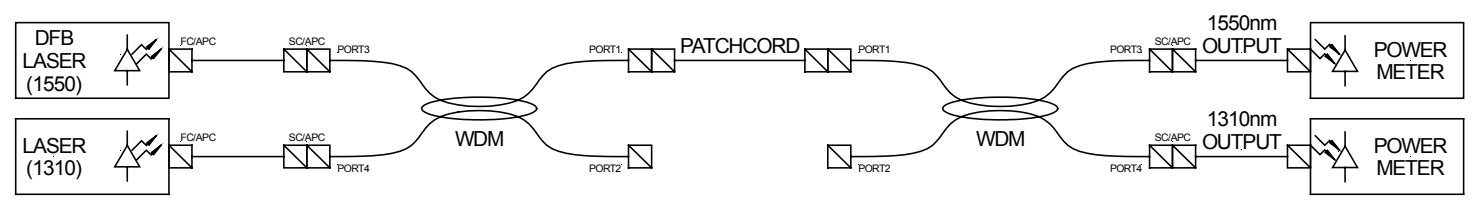

Figure 6: Basic two-channel WDM system 
In order to demonstrate the effects of multiplexing at each point in the system the lasers are then modulated with different signals. Photoreceivers are used to examine the output waveforms after multiplexing and demultiplexing on a suitable oscilloscope. The system and typical results are shown in Figure 7.

MULTIPLEXED SIGNALS

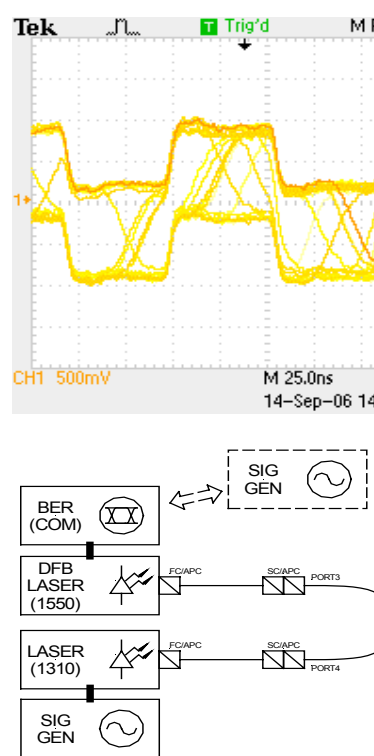

DEMULTIPLEXED SIGNALS

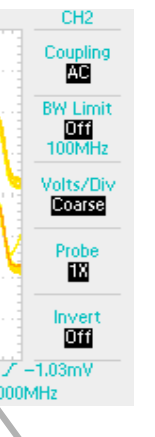

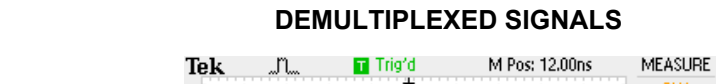

Tek Nh T Trig'd M Pos: 12.00ns MEASUR
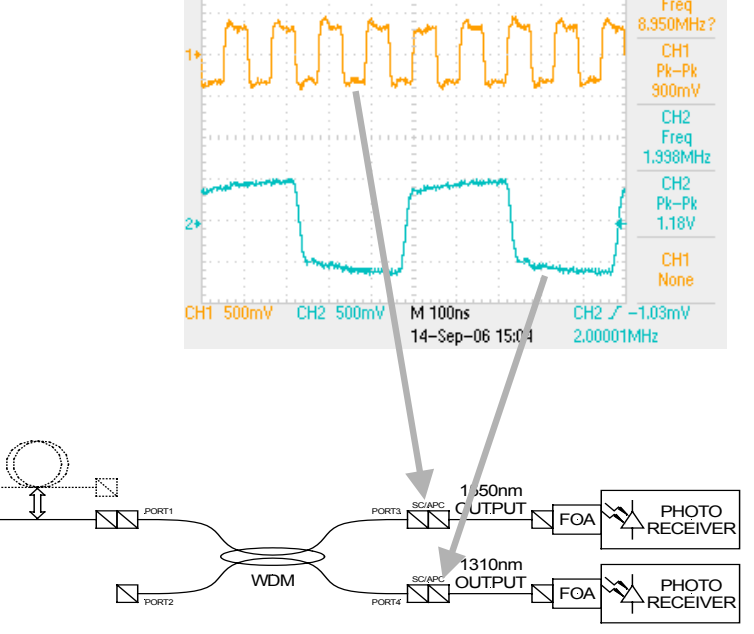

Figure 7: WDM demonstration

\subsubsection{Characteristics of Optical Fibre}

The channel in any communications system comprises every element between the output of the transmitter and the input to the receiver. In an optical system the channel comprises the optical fibre cable plus a few connectors and/ or splices, the receiver and transmitter interfaces (i.e. the terminations) and, in very long distance links, repeaters. The properties of the channel have a strong influence on the performance of the complete system. Digital signals transmitted by the channel are degraded by power loss (attenuation) and pulse spreading (dispersion) in the cabled optical fibre and additional losses are incurred at the splices, connectors and terminations. These effects in turn have a significant bearing on the maximum link length and bit rate.

OptoSci's ED-COM, Fibre Optic Communications, and BER(COM) kits examine these effects in detail using LED and laser sources at wavelengths around $800 \mathrm{~nm}$ and multimode fibre $[3,4]$. With optical communications systems using $800 \mathrm{~nm}$ sources and multimode fibre the attenuation and dispersion effects are larger than at $1310 \mathrm{~nm}$ and $1550 \mathrm{~nm}$ and, with appropriate educator kit design, enable dispersion measurements to be made with standard laboratory equipment. However, the general concepts demonstrated at $800 \mathrm{~nm}$ are equally applicable to state of the art long haul, high capacity fibre links operating at $1310 \mathrm{~nm}$ and $1550 \mathrm{~nm}$. In order to expand upon the experiments in the ED-COM and BER(COM) kits and investigate some of the characteristics of higher capacity fibre links, an experimental section was included in the ED-WDM: 1310/1550 WDM Systems extension examining some attenuation and dispersion phenomena at $1310 \mathrm{~nm}$ and $1550 \mathrm{~nm}$.

The students start with a simple attenuation measurement of a fibre reel with a nominal length of $4.4 \mathrm{~km}$ using the techniques described in the previous sections. An estimate of the fibre length is then made by applying an impulse modulation to the laser and measuring the time of flight. These measurements give typical attenuation co-efficients for the fibre of $0.22 \mathrm{~dB} / \mathrm{km}$ at $1550 \mathrm{~nm}$ and $0.38 \mathrm{~dB} / \mathrm{km}$ at $1310 \mathrm{~nm}$ - agreeing well with manufacturer specifications. This should emphases to the student that $1550 \mathrm{~nm}$ is the lower-loss transmission wavelength.

The important topic of chromatic (intramodal) dispersion is then investigated by examining the transmission of the two wavelengths over various lengths of singlemode fibre. An elegant way of simulating different transmission lengths is by using a ring resonator arrangement as shown in Figure 8(a). The ring resonator is formed simply by connecting the coupled arm (Port 4) and second input (Port 2) of a 50/50 coupler with the fibre reel. 


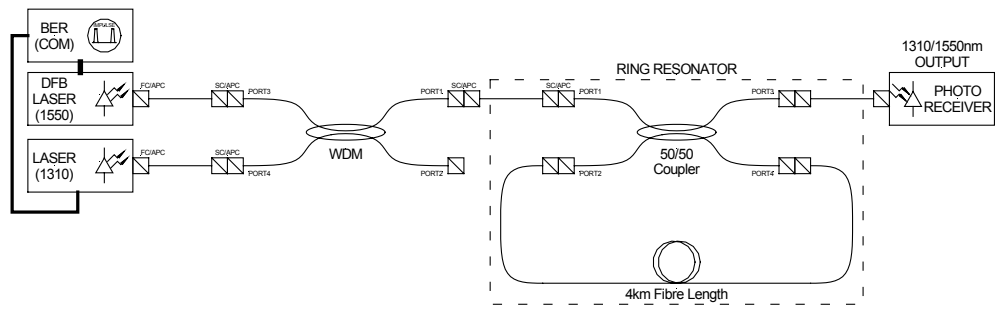

(a)

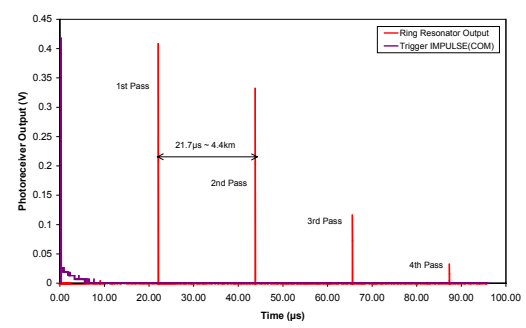

(b)

Figure 8: Fibre chromatic dispersion measurements with ring resonator at $1310 \mathrm{~nm} \& 1550 \mathrm{~nm}$

Applying an impulse modulation to the lasers with this optical arrangement results in a series of pulses (of decreasing size) appearing at the receiver with time delays corresponding to integer multiples of the cavity length (nominally $0,4.4,8.8,13.2,17.6 \mathrm{~km}$ ) as is shown in Figure 8(b).

Taking a closer look at the output after each pass, as shown in Figure 9, demonstrates the effects of chromatic dispersion with the $1550 \mathrm{~nm}$ pulse lagging the $1310 \mathrm{~nm}$ by approximately $9.5 \mathrm{~ns}$ every pass $(4.453 \mathrm{~km})$. This illustrates the possibilities of pulse spreading caused by dispersion over long distances in optical fibre transmission channels.

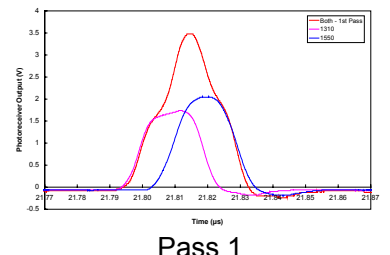

ass 1

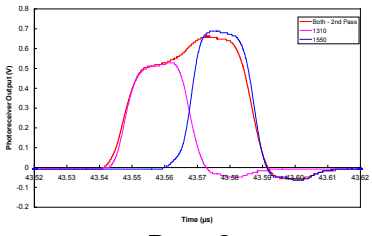

Pass 2

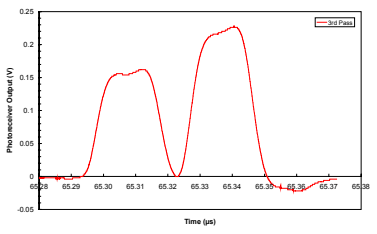

Pass 3

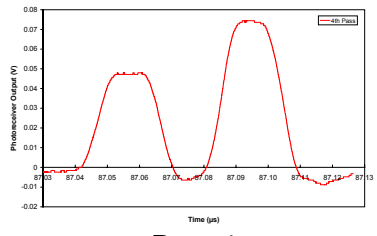

Pass 4

Figure 9: Increasing separation of 1310 \& 1550 pulses after each pass round the ring resonator

\subsection{DWDM Systems}

With the ED-WDM: DWDM Systems extension students are expected to perform the investigation of practical DWDM systems. The following tasks are carried out:

- The examination of a two channel WDM system

- The investigation of WDM System cross-talk

- Examination of the effects of wavelength drift on WDM System performance particularly crosstalk

- Influence of system cross-talk on the Eye Diagram / BER in WDM Systems

The module provides a second DFB laser, operating at a channel adjacent to the original laser, dual InGaAs photoreceivers, a variable optical attenuator (VOA) and an additional OADM (at the adjacent channel wavelength).

In a manner similar to the 1310/1550nm WDM systems kit, this extension demonstrates the fundamental ability of the OADM components to efficiently multiplex and demultiplex signals onto a single optical fibre channel.

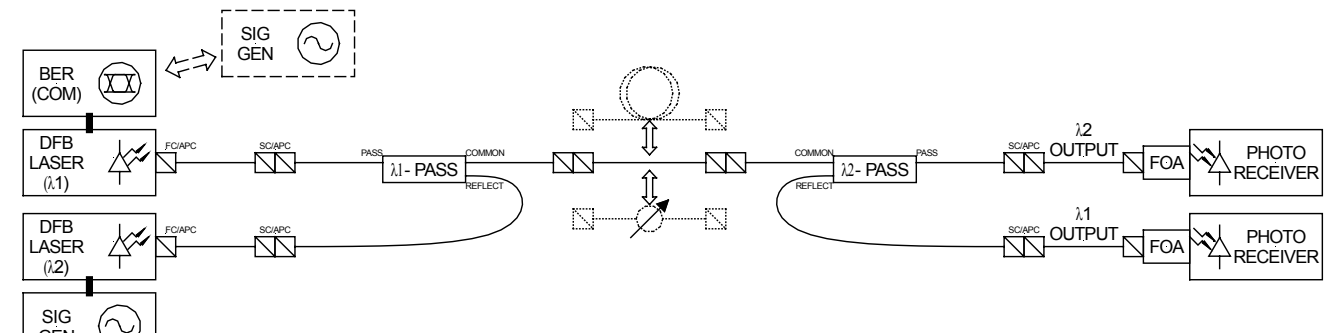

Figure 10: Two-channel DWDM system 
The system constructed by the students is shown in Figure 10 with the basic results identical to the oscilloscope trace shown in Figure 7.

The major difference from the $1310 / 1550$ set-up being highlighted is the $0.8 \mathrm{~nm}$ wavelength separation of the dense WDM channels. The adjacent nature of the channels used can then be used to demonstrate the effects of crosstalk on DWDM systems. Using the experimental set-up shown in Figure 10, the students are directed to detune the wavelength of one lasers from its centre wavelength towards the adjacent channel and examine the effect on the output waveforms. Figure 11 shows the increasing levels of crosstalk appearing on the $1549.32 \mathrm{~nm}$ output port as the $1550.12 \mathrm{~nm}$ laser is tuned to $1549.9 \mathrm{~nm}$.

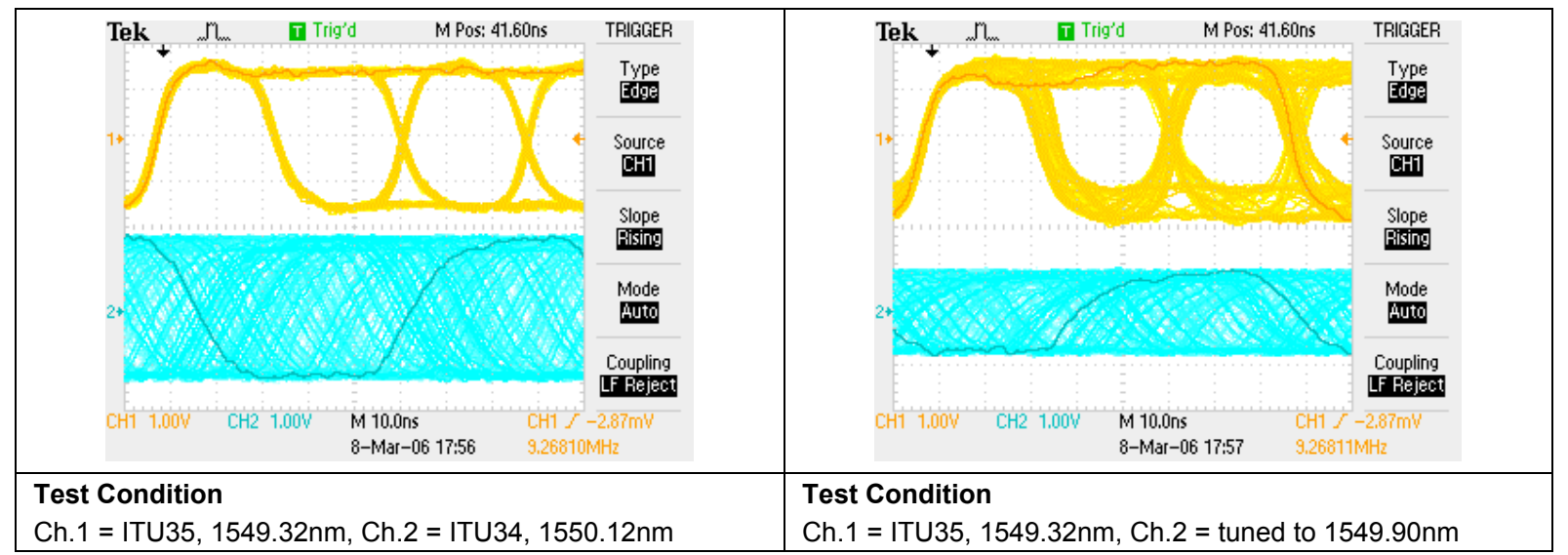

Figure 11: Demonstration of Crosstalk on a DWDM system

A second possible DWDM scenario is then examined. In some WDM systems, Channels may be added and dropped at various points along the optical link. Figure 12 shows a system where a second channel is added at some considerable distance from the Channel 1 input (simulated by high attenuation, $\approx 25 \mathrm{~dB}$, produced by a variable optical attenuator of the Channel 1 signal) and then the Channel 1 signal is dropped a short distance beyond that. The students are then asked to experimentally investigate this type of system in which a strong signal is present at the drop point for a weak signal. In particular they are asked to investigate the effects of wavelength drift in the Channel 2 laser source resulting in crosstalk.

This arrangement can best be studied by using comparison of eye-diagrams and bit-error-rate (BER) analysis of the system operation as the laser wavelength is detuned. The OptoSci BER(COM) kit [4] provides a ready means to carry out this analysis and hence is recommended as a possible add-on. However any suitable PRBS generator may be used (directions for the use of external signal generators are provided in the technical appendices of the literature support).

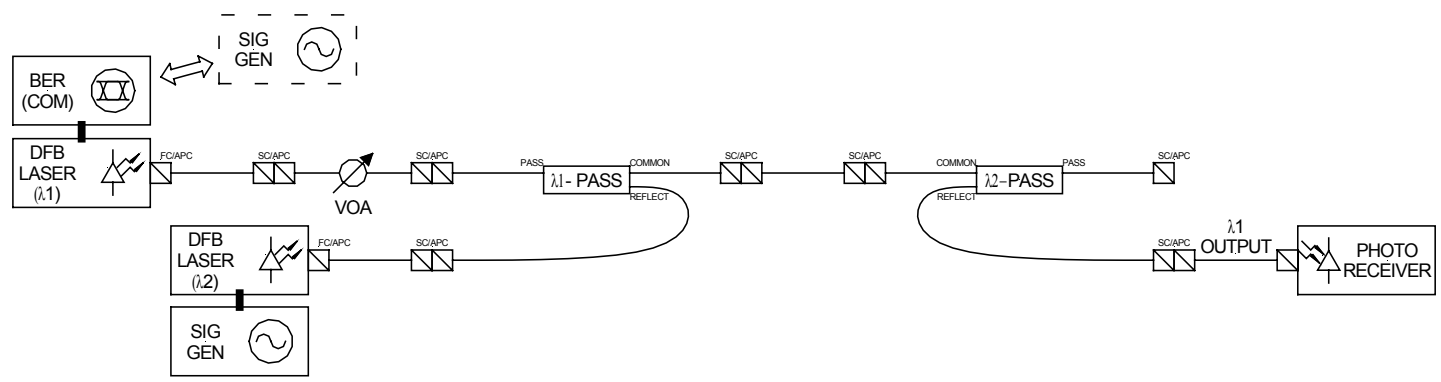

Figure 12: Crosstalk demonstration by adding a strong signal on $\lambda_{2}$ (modulated) to a weak signal on $\lambda_{1}$ (PRBS). The receiver is looking at the weak $\lambda_{1}$ signal with crosstalk from $\lambda_{2}$. 
The students should find that there is a noticeable increase in the noise levels present on the output trace with an associated closing of the eye. This is due to the OADM configuration where channels are more susceptible to crosstalk as the effective isolation is reduced due to the large difference in power levels from the new add channel relative to the low level of the original (attenuated) signal. Using the BER(COM) and its accompanying software to allows analysis of the eye-diagrams and estimated the BERs, a typical set of results (again provided in the instructors supplement) is presented in Table 1 .

\begin{tabular}{|c|c|c||}
\hline Channel 1 (nm) & Channel 2 (nm) & BER (Channel 1) \\
\hline \hline 1549.32 & 1550.12 & $1.00 \times 10^{-11}$ \\
\hline 1549.52 & 1550.12 & $3.30 \times 10^{-10}$ \\
\hline 1549.54 & 1550.12 & $1.30 \times 10^{-09}$ \\
\hline 1549.56 & 1550.12 & $3.10 \times 10^{-08}$ \\
\hline 1549.58 & 1550.12 & $6.30 \times 10^{-07}$ \\
\hline 1549.60 & 1550.12 & $8.69 \times 10^{-06}$ \\
\hline 1549.62 & 1550.12 & $8.00 \times 10^{-05}$ \\
\hline
\end{tabular}

Table 1: BER results for DWDM system.

The performance of the system can be seen to degrade and bit error rates increase rapidly in the configuration shown in Figure 12 and hence the control and accuracy of the laser operating wavelength becomes critical.

\subsection{Bragg Gratings}

The ED-WDM: Bragg Gratings extension concentrates on the topic of fibre Bragg Grating (FBG) Sensors investigating the effects of temperature and examining possible uses as temperature sensors. The kit includes a Bragg grating on a temperature controlled mount and a thermo-electric-cooler (TEC) module.

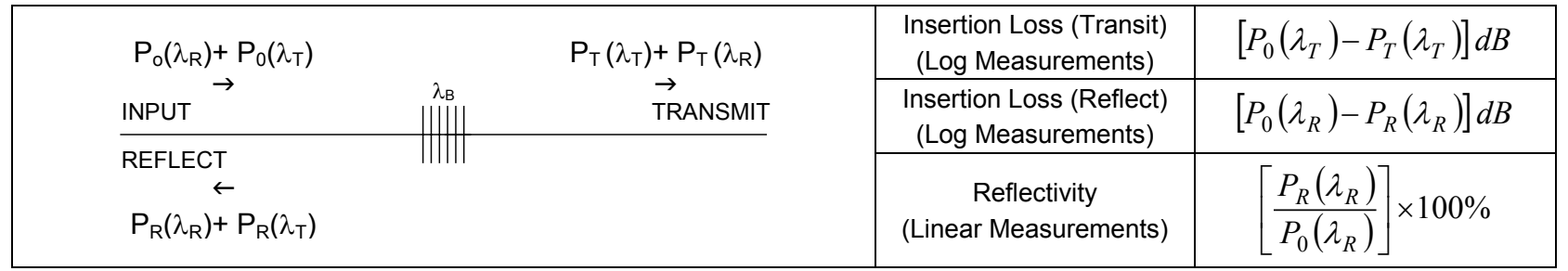

Figure 13: FBG definitions

In order to perform the basic characterisation of a fibre Bragg the students are directed to use the experimental set-up shown in Figure 14. The insertion of the circulator is required to provide a measurement path for the reflected wavelength and to eliminate a return path to the laser source. Clear instruction is provided to ensure the resultant power levels are corrected for the additional power drops associated with passes through the circulator.

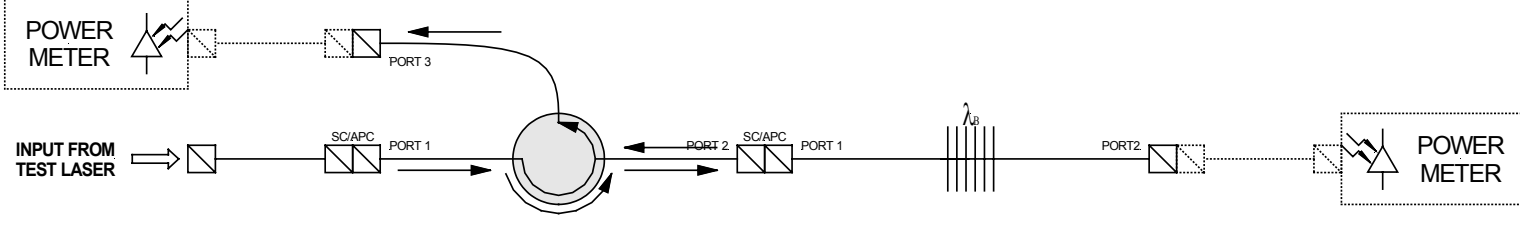

Figure 14: Fibre Bragg grating characterisation

As mentioned earlier the response of the Bragg is very narrowband hence the use of the $\lambda$-scan software is recommended to achieve a full measurement set. A typical pair of responses for the transmit and reflect conditions is shown in Figure 15(a). Figure 15(b) shows how the transmission of the Bragg grating changes as the temperature is varied using the TEC module. 


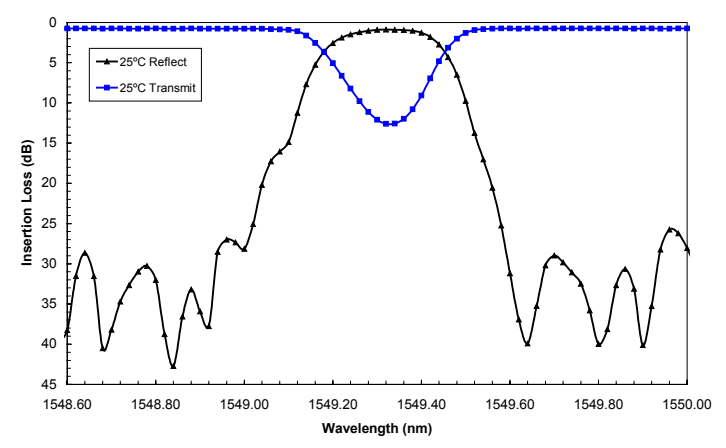

(a)

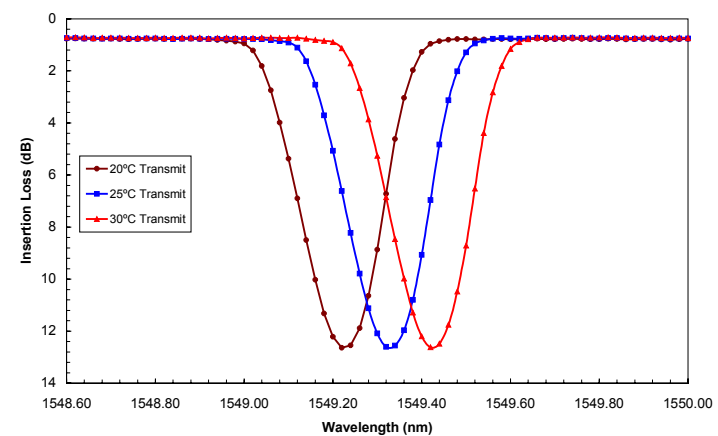

(b)

Figure 15: Bragg Grating spectral responses, (a) transmission and reflection at $25^{\circ} \mathrm{C}$, and (b) transmission at $20^{\circ} \mathrm{C}, 25^{\circ} \mathrm{C}$ and $30^{\circ} \mathrm{C}$

From Figure $15(\mathrm{~b})$ the temperature co-efficient can be calculated as $20 \mathrm{pm} /{ }^{\circ} \mathrm{C}$. The changes in grating temperature trigger corresponding variations in the period of the grating and thus the wavelength of light that is reflected. This Bragg reflection shift makes it straightforward to use Bragg gratings to track variations in environmental parameters such as temperature and strain. The fact that multiple Bragg gratings can be written within an optical fibre also make these sensors amenable to direct and non-intrusive integration within the body of composite materials used in civil structures, aerospace platforms, etc. in order to provide detailed structural health monitoring information.

\section{Conclusions}

In this paper we have described a suite of laboratory based educational packages which has been developed to allow students to explore and examine the concepts, components and systems used in fibre optic WDM and experimentally demonstrate the effects of system crosstalk and chromatic dispersion. The packages provide the theoretical background of the operation of WDM components, measurement techniques and concepts of WDM systems before providing the hardware to allow the student to perform an experimental investigation.

Throughout the packages the emphasis is not only in presenting students with the theoretical background but also in offering an understanding of the practical side of fibre optic components and systems. Thus on completion of the package the student should have attained a good working knowledge of the components and be familiar with the operation of WDM systems.

The modular format adopted for the packages enables the instructor to target the specific areas or level desired to suit the students. This format has the additional benefit of allowing the instructor to build up the systems from the basics to the more advanced topics as the educational requirements demand and teaching budgets permit.

\section{References}

1. See www.optosci.com for extensive additional information on OptoSci's range of photonics educator kits.

2. W. Johnstone, B. Culshaw, D. Moodie, I. Mauchline and D. Walsh, "Photonics laboratory teaching experiments for scientists and engineers", $7^{\text {th }}$ International conference on Education and Training in Optics and Photonics (ETOP), Singapore, 2001, Paper 304 and SPIE Proceedings 4588, 2002.

3. W. Johnstone, B. Culshaw, D. Walsh, D. Moodie and I. Mauchline, "Photonics laboratory experiments for modern technology based courses", IEEE Proceedings: Special issue on Electrical and Computer Engineering Education, pp41-54, 1999.

4. D. Walsh, D. Moodie, I. Mauchline, S. Conner, W. Johnstone, B Culshaw, "Practical Bit Error Rate Measurements on Fibre Optic Communications Links in Student Teaching Laboratories", $9^{\text {th }}$ International Conference on Education and Training in Optics and Photonics (ETOP), Marseille, France, Paper ETOP021, 2005. 